\title{
PRESENTING THE BRAZILIAN JOURNAL OF MEDICINE AND HUMAN HEALTH
}

\begin{abstract}
Medicine is an ancient science. Archeological discoveries have suggested that the Egyptians performed surgeries that involved specific knowledge of human anatomy, probably acquired through the process of corpse mummification, which made it necessary to remove the entrails. Hippocrates, even today considered the "Father of Medicine", played a relevant role in the understanding of diseases, because he was capable of demonstrating the relationship between "symptoms" and the various pathologies. After this, there have been important names in the history of Medicine, for example, William Harvey, with the escription of the circulatory system, Louis Pasteur with the discovery that bacteria are associated with a large number of diseases, Maria Skodowska Curie, "Madame Curie", the first woman to win a Nobel Prize, who, in collaboration with her husband Pierre Curie, "invented" radioactivity and discovered new radioactive elements - thorium, polonium and radium. Their work allowed the development of medical applications of $X$-rays, and consequently the advancements of diagnostic imaging in medicine.
\end{abstract}

Contemporary medicine is a complex science that has an increasingly sophisticated diagnostic arsenal at its disposal, with reference to anatomic and functional knowledge of the human body; laboratory exams that reveal our immunologic, genetic and even ethnic profiles, and expose our vulnerable anatomy in precise detail. Innumerable drugs are capable of curing, controlling, and if we consider vaccines, even preventing diseases. It is demanded of doctors and other health professionals, who envisage not only "curing" disease, but understanding the patient from his/her complex biopsychosocial aspect, to have a humanized professional approach integrated with multidisciplinary practice, and that these attitudes must be based on consolidated scientific evidences.

The Brazilian Journal of Medicine and Human Health, is the scientific journal of the Bahiana School of Medicine and Public Health, maintained by the Foundation for the Development of Sciences, a private, non-profit organization in Salvador, Bahia. Published in English, it opens the perspective for publications of great scientific value, which may contribute to knowledge about human health, spanning the entire scope of its diversity and particularities. Therefore, this semestral periodical offers to all professionals, who aim to divulge their knowledge about the health/disease complex.

The first 2015 number of Brazilian Journal of Medicine and Public Health has four kinds of interesting items: an original article on socio-demographic characteristics of revascularized patients in a public hospital in Brazil, a brief report on current state and perspectives of medical practice, education and research in Brazil, a review of hepatitis $C$ infection and one case report of mesenteric vasculitis in lupus. It is expected that all of these papers can contribute to increase the scientific knowledge of our readers.

Ana Marice Ladeia

Editor 